

\title{
Stochastic processes of limited frequency and the effects of oversampling
}

\author{
D.S.G. Pollock \\ Department of Economics, University of Leicester, \\ University Road, Leicester LE1 7RH, U.K.
}

\begin{abstract}
Discrete-time ARMA processes can be placed in a one-to-one correspondence with a set of continuous-time processes that are bounded in frequency by the Nyquist value of $\pi$ radians per sample period. It is well known that, if data are sampled from a continuous process of which the maximum frequency exceeds the Nyquist value, then there will be a problem of aliasing. However, if the sampling is too rapid, then other problems will arise that may cause the ARMA estimates to be severely biased. The paper reveals the nature of these problems and it shows how they may be overcome.
\end{abstract}

Key words: ARMA Modelling, Stochastic Differential Equations, Frequency-Limited Stochastic Processes, Oversampling

\section{Introduction}

Modern digital communications depend on data sampled at regular intervals from continuously varying signals. The so-called sampling theorem defines the circumstances in which the continuous trajectory of the signal can be recovered from the discrete data. Although this crucial theorem is commonly attributed to Nyquist $(1924,1928)$ and to Shannon (1949), there are others who can reasonably lay claim to its discovery, as Luke (1999) has observed.

The sampling theorem indicates that, if at least two observations are made in the time that it takes the signal component of highest frequency to complete a single cycle, then a continuous signal can be reconstructed perfectly from the sampled sequence. In effect, the theorem poses a limit to the frequencies that can be captured by the sampled data. The limit is the so-called Nyquist frequency of $\pi$ radians per sampling interval.

Email address: stephen_pollock@sigmapi.u-net.com (D.S.G. Pollock). 
Sampling at an insufficient rate, also described as undersampling, leads to a problem of aliasing whereby elements of the signal of frequencies in excess of the Nyquist limit are confounded with elements of frequencies that lie within the observable range. A good account of how this arises has been provided by Oppenheim et al. (1983, Ch 8.), albeit that there are many other accessible sources.

The problem of aliasing is sometimes present in moving cinema pictures, which are created from the rapid projection of a succession of images that capture instants in the trajectories of moving objects. The problem is familiar to a generation who watched Western movies and who noticed the seemingly slow and sometimes retrograde motion in the depiction of the rapidly rotating wheels of a fleeing stagecoach.

The sampled data can be used to construct models of the processes generating the signals. Often, it is sufficient to model the relationships subsisting in the sampled data, with only a passing reference to the underlying continuous process. This may be achieved by fitting an autoregressive moving-average (ARMA) model to the data. However, dynamic systems may be described better by differential equations operating in continuous time. Therefore, the issue arises of how to make the translation from discrete-time data to a continuoustime model.

The usual methods for constructing continuous-time models from the discrete data are fraught with difficulties, and they depend on assumptions that may conflict with the evident properties of the signals. (A recent survey of these methods has been provided by Garnier and Wang 2008.)

The usual assumption that underlies the estimation of stochastic differential equations is that their primum mobile or forcing function is a whitenoise process consisting of a continuous stream of infinitesimal increments of a stochastic Wiener process. Such a white-noise process, which is everywhere continuous but nowhere differentiable, is unbounded in frequency. Thus, an assumption is commonly adopted that implies the inevitability of aliasing and that seems to preclude the possibility of estimating a model that faithfully represents the continuous process. However, the problems are not always so severe.

It can be shown that, if the poles of the transfer function that maps from the white-noise forcing function to the signal have frequency arguments that fall within the Nyquist range, then the stochastic differential equation can be estimated consistently. (See, for example, Pandit and Wu, 1983, Ch 7.) Also, it may be observed that if the transfer function of the model strongly attenuates the higher frequencies, then the assumption that the frequencies of the forcing function are unbounded is of limited significance.

Notwithstanding these easements in the estimation of stochastic differential equations, it is sometimes important to recognise the frequency limits of a stochastic forcing function. It is the purpose of this paper to highlight the problems that can arise when the frequency limit of the forcing function, and therefore of the signal, is less than the Nyquist value. Problems can then arise 
both with the discrete-time autoregressive moving-average model and with the corresponding continuous-time stochastic differential equation.

In some circumstances, it should be possible to reduce the rate of sampling so that the Nyquist frequency no longer exceeds the maximum frequency of the signal. Even when is it is not possible to vary the sampling rate directly, it may be possible to reconstitute the continuous signal in the manner indicated by the sampling theorem.

Then, it is possible to generate the sample points that would arise from resampling the continuous signal at an arbitrary rate. Thus, a sample can be obtained that is attuned to the maximum frequency within the signal. The relevant procedure will be described in this paper. However, a example will be presented for which it is sufficient to subsample the data by taking one in every four points.

The primary purpose of this paper is to illustrate the problems of overrapid sampling, also described as oversampling, and to show how to overcome them. Nevertheless, it will be necessarily to deal more generally with the theory of autoregressive moving-average models and of the corresponding stochastic differential equations.

The plan of the paper is as follows. In section 2, the problems of oversampling are illustrated in the context of a macroeconomic data sequence. In section 3, an account is given of the essential sampling theorem; and it is shown how it can be adapted to finite data sequences. Section 4 provides the theory of frequency-limited continuous-time processes that bear a one-to-one correspondence with discrete-time ARMA models, and section 5 explains the empirical findings of section 2.

With these results in hand, it will be show, in section 6 , how it is possible, by means of a process of re-sampling, to obtain appropriate estimates of frequency-limited ARMA processes by means of the usual estimators of discrete-time ARMA models. Thereafter, it remains to describe, in section 7 , how a linear stochastic differential equation (LSDE) may be fitted to the frequency-limited data.

\section{The Effects of Oversampling}

The typical frequency-limited spectral structure can be illustrated by a data sequence that requires only a simple method of detrending. Figure 1 shows the deviations from an interpolated trend of the logarithms of U.S. quarterly gross domestic product (GDP) for the period 1968-2007. The trend has been calculated using the filter of Hodrick and Prescott $(1980,1997)$ with the smoothing parameter set to the value of 1600. (The filter in question is also attributable to Leser 1961.)

Figure 2 shows the periodogram of the deviations. It will be seen that the essential spectral structure extends no further than the frequency of $\pi / 4$ radians per quarter. The remainder of the periodogram comprises what may 


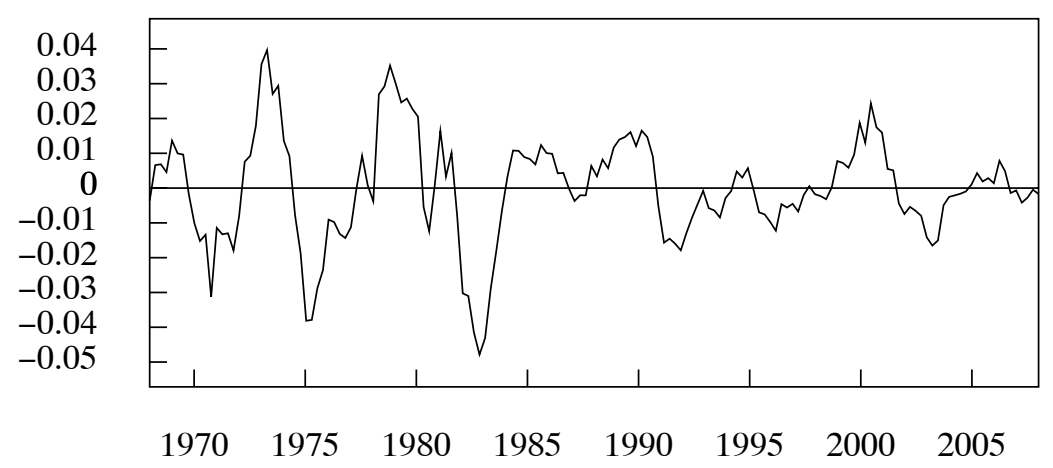

Fig. 1. The deviations of the logarithmic quarterly index of real US GDP from an interpolated trend. The observations are from 1968 to 2007. The trend is determined by a Hodrick-Prescott (Leser) filter with a smoothing parameter of 1600 .

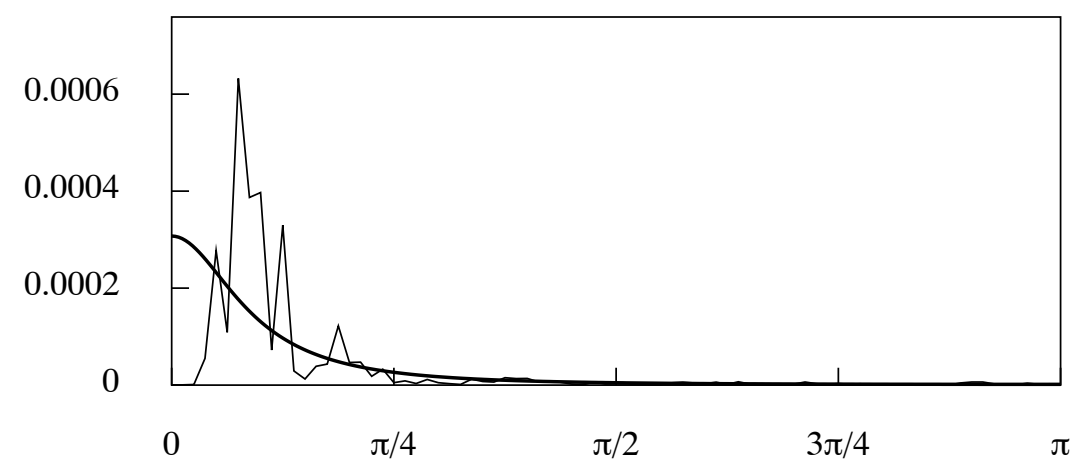

Fig. 2. The periodogram of the data points of Figure 1 overlaid by the parametric spectral density function of an estimated regular $\mathrm{AR}(2)$ model.

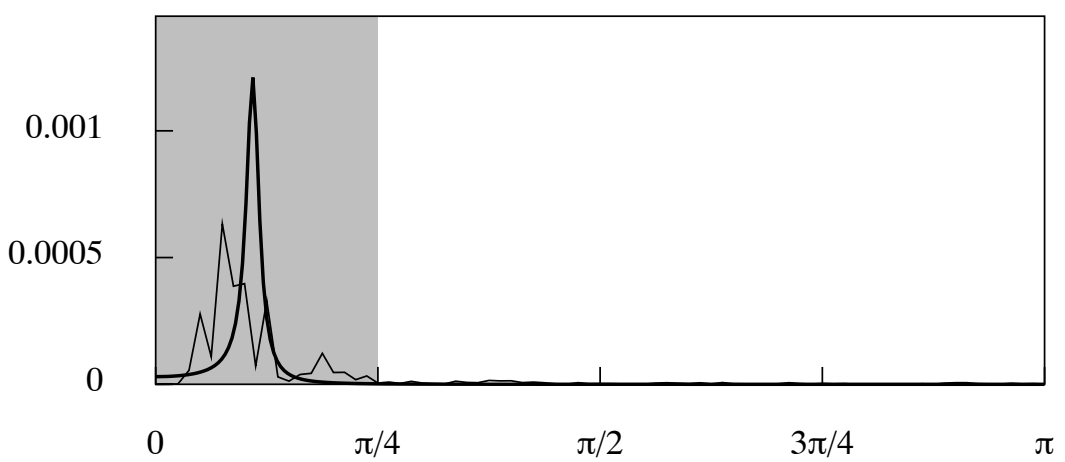

Fig. 3. The periodogram of the data points of Figure 1 overlaid by the spectral density function of an $\mathrm{AR}(2)$ model estimated from frequency-limited data.

be described as a dead space that contains only the traces of minor elements of noise. It is notable that the detrending of the data has imposed a zero at the zero frequency.

One might hope to characterise the dynamic properties of the US GDP process by fitting an ARMA model of relatively low orders. Thus, a second- 
order autoregressive $\mathrm{AR}(2)$ model with complex conjugate roots or poles within the autoregressive polynomial should serve to represent the dynamics. The argument of the complex roots should characterise the angular velocity of the process, or equivalently, the length of its cycles, and their modulus should characterise its damping properties.

However, when such a model is fitted to the data, it transpires, almost invariably, that the roots of the autoregressive polynomial are real-valued. (For a testimony to this, see Pagan 1997.) Figure 2 shows such an outcome, which can be discerned from the shape of the parametric spectral density function, or "spectrum", of the estimated model.

A reasonable recourse in the face of such difficulties is to employ an estimator in which greater weight is placed on the low-frequency elements of the data than on the high-frequency elements. If the interest is confined to the business cycles, then it is reasonable to attribute weighs of unity to the elements within the range of the business cycle frequencies and to attribute weights of zero to those elements that lie outside the range. This can achieved by applying an ordinary autoregressive Yule-Walker method-of-moments estimator to data that are limited to the low-frequency range. Such data can be created by applying a perfect lowpass filter to the original data. (For a description of such a filter, see Pollock, 2009.)

The consequence of applying an unrestricted estimator to data that are strictly frequency-limited will be to create an estimated autoregressive polynomial in which the complex roots approach the perimeter of the unit circle. The effect will be to misrepresent the damping properties of the process. Figure 3 illustrates this outcome.

The only difference between the circumstances depicted in Figures 2 and 3 concerns an almost imperceptible noise contamination that is found throughout the interval $(\pi / 4, \pi]$ in the original data. The ratio of the cumulated values of the periodogram ordinates that lie in this frequency interval to the cumulated total of periodogram ordinates over the interval $[0, \pi]$ is 0.0752 , or less than 8 percent. Whereas this noise is affecting the estimates that give rise to the parametric spectrum of Figure 2, it has been eliminated from estimation of the spectrum of Figure 3.

A full explanation of these effects, which will be provided in section 5 , must await a detailed analysis of the sampling process and of frequency-limited stochastic processes.

\section{The Sampling Process}

The sampling theorem of Shannon and Nyquist establishes that, if the maximum frequency within a continuous square-integrable function is $\omega_{c} \leq \pi$ radians per sample interval, which is taken to be the unit of time, then all of the information within the function can be conveyed via a sequence of values sampled regularly from the function at intervals of $\pi / \omega_{c}$ units of time. (See 


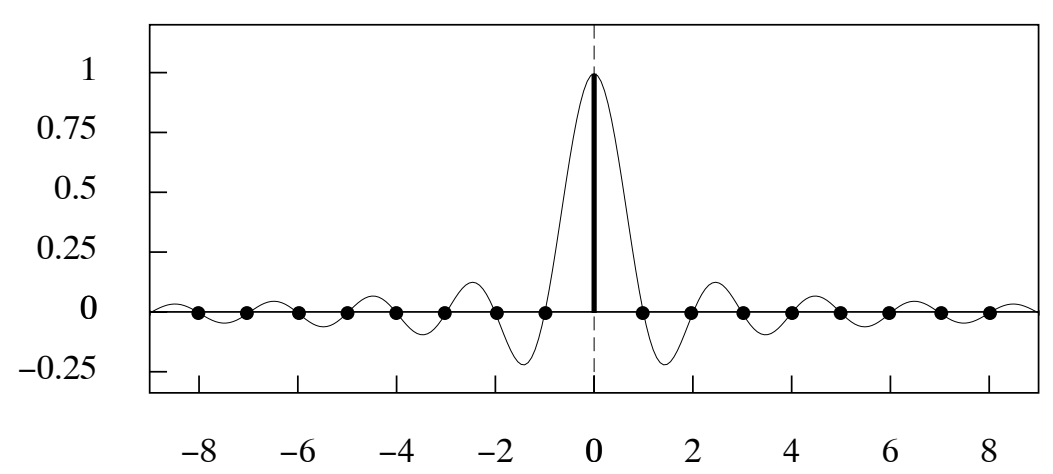

Fig. 4. The sinc function wave-packet $\varphi(t)=\sin (\pi t) / \pi t$ comprising frequencies in the interval $[0, \pi]$.

Shannon, 1949.)

The theorem implies that, if samples are taken at unit intervals, then the maximum frequency that can be detected is at $\pi$ radians per interval, which is the so-called Nyquist frequency. Moreover, if the underlying function contains frequencies in excess of $\pi$, then, via the process of aliasing, these will be confounded with frequencies that lie within the Nyquist frequency interval $[0, \pi]$.

The interval $[0, \pi]$ is appropriate to the case where the function in question is expressed in terms of sines and cosines. When it is expressed, equivalently, in terms of complex exponential functions, the Nyquist frequency range becomes $[-\pi, \pi]$. In the case of a function with a maximum frequency of $\pi$ radians per sample interval, it will be possible, in theory, to reconstitute the continuous function from its sampled values by associating to each of them a so-called sinc function kernel scaled by that value.

The sinc function $\varphi(t)$ is the Fourier transform of a frequency-domain rectangle $\varphi(\omega)$ supported on the interval $[-\pi, \pi]$; and it is just a sine function to which a hyperbolic taper has been applied:

$$
\varphi(t)=\frac{\sin (\pi t)}{\pi t}=\frac{1}{2 \pi} \int_{-\pi}^{\pi} e^{\mathrm{i} \omega t} d \omega .
$$

This is illustrated in Figure 4.

The following expression for the continuous function is derived by applying a sinc function kernel to each element of the sequence $\left\{x_{k} ; k=0, \pm 1, \pm 2, \ldots\right\}$ :

$$
x(t)=\sum_{k=-\infty}^{\infty} x_{k} \frac{\sin \{\pi(t-k)\}}{\pi(t-k)}=\sum_{k=-\infty}^{\infty} x_{k} \varphi(t-k) .
$$

Here, $t \in \mathcal{R}$ is to be regarded as a continuous index of time. The formula indicates that there is a one-to-one correspondence between continuous functions supported on the Nyquist frequency interval and their sampled ordinates taken at unit intervals in time. 
Although the Shannon-Nyquist theorem is proved in relation to a square integrable function, it is clear that the construction above can be extended to encompass stationary stochastic processes. Thus, it might be assumed that the doubly-infinite sequence $\left\{x_{k} ; k=0, \pm 1, \pm 2, \ldots\right\}$ is generated by an ordinary autoregressive moving-average process.

The sequence of sinc functions $\varphi(t-k) ; k \in \mathcal{Z}=\{0, \pm 1, \pm 2, \ldots\}$ constitutes an orthogonal basis for the set of all continuous analytic functions that lie within the Nyquist frequency interval. To show this, let $\varphi(\omega)$ be the Fourier transform of $\varphi(t)$ and consider the following autoconvolution:

$$
\begin{aligned}
\int_{t} \varphi(t) \varphi(\tau-t) d t & =\int_{t} \varphi(t)\left\{\frac{1}{2 \pi} \int_{\omega} \varphi(\omega) e^{\mathrm{i} \omega(\tau-t)} d \omega\right\} d t \\
& =\frac{1}{2 \pi} \int_{\omega} \varphi(\omega)\left\{\int_{t} \varphi(t) e^{-\mathrm{i} \omega t} d t\right\} e^{\mathrm{i} \omega \tau} d \omega \\
& =\frac{1}{2 \pi} \int_{\omega} \varphi(\omega) \varphi(\omega) e^{\mathrm{i} \omega \tau} d \omega=\varphi(\tau) .
\end{aligned}
$$

The symmetry of $\varphi(t)$ allows us to write $\varphi(\tau-t)=\varphi(t-\tau)$, whereas the idempotency of $\varphi(\omega)$ gives $\varphi^{2}(\omega)=\varphi(\omega)$. Together, these two conditions indicate that $\varphi(t)$ is its own autocorrelation function. Therefore, the condition

$$
\varphi(t)=0 \quad \text { for } \quad t \in\{ \pm 1, \pm 2, \ldots\}
$$

which is manifest in the formula of (1) and in Figure 3., indicates that sinc functions separated by integer distances are mutually orthogonal.

When the set of sinc functions $\{\varphi(t-k) ; k \in \mathcal{Z}\}$ at unit displacements are sampled at the integer values of $t$, the result is nothing but the set of unit impulses at the integer points. This constitutes a basis for the set of all sequences defined over the set of integers.

The reconstruction or interpolation of a function in the manner suggested by the sampling theorem is not possible in practice, because it requires summing an infinite number of sinc functions, each of which is supported on the entire real line. Nevertheless, a continuous frequency-limited periodic function, defined on a finite interval, can be reconstituted from a finite number of wrapped or periodic sinc functions, which are Dirichlet kernels by another name. The Dirichlet kernel is obtained by sampling the frequency-domain rectangle of the sinc function.

Consider a continuous function $x(t)$ defined the interval $[0, T)$. Such a function can be regarded as a single cycle of a periodic or circular function such that $x(t)=x(t+T)$; and, therefore, it has a Fourier series expansion in terms of the complex exponential functions $\exp \left(i \omega_{j}\right)$, where $\omega_{j}=2 \pi j / T$ is the $j$ th Fourier frequency. If the function $x(t)$ is bounded by the Nyquist frequency, then the relevant expression for the series expansion of $x(t)$, together with the 
inverse transformation, which provides the Fourier coefficients $\xi_{j}$, is

$$
x(t)=\sum_{j=0}^{T-1} \xi_{j} e^{\mathrm{i} \omega_{j} t} \longleftrightarrow \xi_{j}=\frac{1}{T} \sum_{t=0}^{T-1} x(t) e^{-\mathrm{i} \omega_{j} t} .
$$

Here, $t \in \mathcal{R}$ is considered to be a continuous index of time. When $t=$ $0,1, \ldots, T-1$ is an integer, the equation represents the discrete Fourier transform of the sampled sequence $\left\{x_{t} ; t=0,1, \ldots, T-1\right\}$ together with the inverse transform.

Putting the expressions for the Fourier ordinates at the sample points into the finite Fourier series expansion of the time function and commuting the summation signs gives

$$
x(t)=\sum_{j=0}^{T-1}\left\{\frac{1}{T} \sum_{k=0}^{T-1} x_{k} e^{-\mathrm{i} \omega_{j} k}\right\} e^{\mathrm{i} \omega_{j} t}=\frac{1}{T} \sum_{k=0}^{T-1} x_{k}\left\{\sum_{j=0}^{T-1} e^{\mathrm{i} \omega_{j}(t-k)}\right\} .
$$

The inner summation of the final expression gives rise to the Dirichlet Kernel:

$$
\varphi_{n}^{\circ}(t)=\sum_{t=0}^{T-1} e^{i \omega_{j} t}=\frac{\sin \left(\{(T-1) / 2\} \omega_{1} t\right)}{\sin \left(\omega_{1} t / 2\right)} .
$$

Thus, the Fourier expansion can be expressed in terms of the Dirichlet kernel, which is a circularly wrapped sinc function:

$$
x(t)=\frac{1}{T} \sum_{t=0}^{T-1} x_{k} \varphi_{n}^{\circ}(t-k) .
$$

The functions $\left\{\varphi^{\circ}(t-k) ; k=0,1, \ldots, T-1\right\}$ are appropriate for reconstituting a continuous periodic function $x(t)$ defined on the interval $[0, T)$ from its sampled ordinates $x_{0}, x_{1}, \ldots, x_{T-1}$. However, the periodic function can also be reconstituted by an ordinary Fourier interpolation

$$
x(t)=\sum_{j=0}^{T-1} \xi_{j} e^{\mathrm{i} \omega_{j} t}=\sum_{j=0}^{[T / 2]}\left\{\alpha_{j} \cos \left(\omega_{j} t\right)+\beta_{j} \sin \left(\omega_{j} t\right)\right\},
$$

where $[T / 2]$ denotes the integral part of $T / 2$ and where $\alpha_{j}=\xi_{j}-\xi_{-j}$ and $\beta_{j}=$ $\mathrm{i}\left(\xi_{j}+\xi_{-j}\right)$ are the coefficients from the regression of the data on the sampled ordinates of the cosine and sine functions at the various Fourier frequencies.

If $t \in\{0,1, \ldots, T-1\}$ is the integer index of discrete time, then the formula of (9) will serve to generate the sampled ordinates. If $t \in[0, T)$ is a continuous real-valued index, then the formula will serve to reconstitute the continuous signal that is presumed to underlie these data. 


\section{Frequency-Limited Stochastic Processes}

A continuous-time autoregressive moving-average process that is supported on the Nyquist interval $[-\pi, \pi]$ may be derived from an ordinary discrete-time process with unit time intervals by associating sinc functions to each of its ordinates. By virtue of the Shannon-Nyquist theorem, there will be a one-to-one correspondence between the discrete-time processes and the frequency-limited continuous-time processes.

Linear stochastic differential equations (LSDE's) that are driven by the increments of a Wiener process are commonly described as CARMA processes. To avoid confusion, these will be described as LSDE processes. An continuoustime ARMA process driven by a frequency-limited of "band-limited" white noise can also be represented by a linear stochastic differential equation. Such a representation will be provided in section 7 .

The continuous-time ARMA process can be derived directly by applying an appropriate filter to a continuous frequency-limited white-noise process supported on the Nyquist interval. The continuous white-noise process is obtained from a train of sinc-function wave packets arriving regularly at unit intervals of time and having amplitudes that are distributed independently and identically in the manner of the ordinates of a discrete-time white-noise process.

It should be observed that the datum $t=0$, from which the discrete points in time are measured, is arbitrary. Any other point on the real line could serve as the datum; and a decomposition of the continuous function will always be available that attributes sinc-function pulses to the sequence of discrete points, measured in unit steps from that datum.

Let $\left\{y_{k} ; k=0, \pm 1, \pm 2, \ldots\right\}$ be the sampled ordinates of the ARMA process and let $\left\{\varepsilon_{k} ; k=0, \pm 1, \pm 2, \ldots\right\}$ be the ordinates of the white-noise forcing function. The corresponding continuous-time functions are

$$
y(t)=\sum_{k=-\infty}^{\infty} y_{k} \varphi(t-k) \text { and } \varepsilon(t)=\sum_{k=-\infty}^{\infty} \varepsilon_{k} \varphi(t-k),
$$

respectively, where $t \in \mathcal{R}$ and $k \in \mathcal{Z}$ and where $\varphi(t)$ is the sinc function kernel. The equation of the continuous-time ARMA process is

$$
\sum_{j=0}^{p} \alpha_{j} y(t-j)=\sum_{j=0}^{q} \beta_{j} \varepsilon(t-j),
$$

where $\alpha_{0}=1$. This has a moving-average representation in the form of

$$
y(t)=\sum_{j=0}^{\infty} \psi_{j} \varepsilon(t-j),
$$

where the coefficients are from the series expansion of the rational function $\beta(z) / \alpha(z)=\psi(z)$. 
If $\varepsilon_{t}=\varepsilon(t)$ and $\varepsilon_{s}=\varepsilon(s)$, with $t, s \in \mathcal{R}$, are elements sampled at arbitrary points from the continuous frequency-limited white-noise forcing function, then their covariance is

$$
C\left(\varepsilon_{t}, \varepsilon_{s}\right)=\sigma_{\varepsilon}^{2} \varphi(t-s)=\gamma_{\varepsilon}(\tau), \quad \tau=t-s,
$$

where $\sigma_{\varepsilon}^{2}$ is the variance parameter. This the autocovariance function of the white-noise process. The result is understood by recognising that $\varepsilon_{s}=\varphi(\tau) \varepsilon_{t}+$ $\eta$, where $\eta$ is uncorrelated with $\varepsilon_{t}$. This result is in accordance with equation (3), which indicates that $\varphi(\tau)$ is its own autocorrelation function.

If $y(t)=\sum_{i} \psi_{i} \varepsilon(t-i)$, and $y(s)=\sum_{j} \psi_{j} \varepsilon(s-j)$, with $t, s \in \mathcal{R}$ and $i, j \in \mathcal{Z}$, then the autocovariance function of $y_{t}=y(t)$ and $y_{s}=y(s)$ is

$$
\begin{aligned}
C\left\{\sum_{i} \psi_{i} \varepsilon_{t-i}, \sum_{j} \psi_{j} \varepsilon_{s-j}\right\} & =\sum_{i} \sum_{k} \psi_{i} \psi_{i+k} C\left(\varepsilon_{t}, \varepsilon_{s-k}\right) ; \quad k=j-i \\
& =\sum_{k} \gamma_{k} \varphi(\tau-k)=\gamma(\tau) ; \quad \tau=t-s
\end{aligned}
$$

where $\gamma_{k}=\sigma_{\varepsilon}^{2} \sum_{j} \psi_{j} \psi_{j+j}$ is the $k$ th autocovariance of the discrete-time process. It can be seen immediately that $\gamma(\tau)=\gamma_{\tau}$, is a discrete-time autocovariance when $\tau$ takes an integer value, and that the continuous-time autocovariance function is obtained from the discrete-time function by sinc-function interpolation.

The autocovariances of a discrete-time ARMA process are the coefficients of the series expansion of the generating function

$$
\gamma(z)=\sigma_{\varepsilon}^{2} \frac{\beta(z) \beta\left(z^{-1}\right)}{\alpha(z) \alpha\left(z^{-1}\right)}
$$

where $\alpha(z)=\alpha_{0}+\alpha_{1} z+\cdots+\alpha_{p} z^{p}$ and $\beta(z)=\beta_{0}+\beta_{1} z+\cdots+\beta_{q} z^{q}$ are the autoregressive and moving-average polynomials respectively. Here, it may be assumed the $p>q$, since this is a necessary condition for the existence of a stochastic differential equation corresponding to the ARMA process.

A recursive procedure for generating the autocovariance of a discrete-time ARMA process has been provided by Pollock (1999). An analytic expression is also available that is based on the following partial-fraction decomposition of the transfer function:

$$
\psi(z)=\frac{\beta(z)}{\alpha(z)}=\frac{d_{1}}{1-\mu_{1} z}+\cdots+\frac{d_{p}}{1-\mu_{p} z} .
$$

Here, $\mu_{1}, \ldots, \mu_{p}$ are the roots of the polynomial equation $\alpha\left(z^{-1}\right)=0$. (It is assumed that there are no repeated roots.) The general analytic expression for the autocovariance function of an ARMA process is

$$
\gamma(\tau)=\sigma_{\varepsilon}^{2} \sum_{i=1}^{p}\left\{\sum_{j=1}^{p} \frac{d_{i} d_{j}}{1-\mu_{i} \mu_{j}}\right\} \mu_{i}^{\tau} .
$$




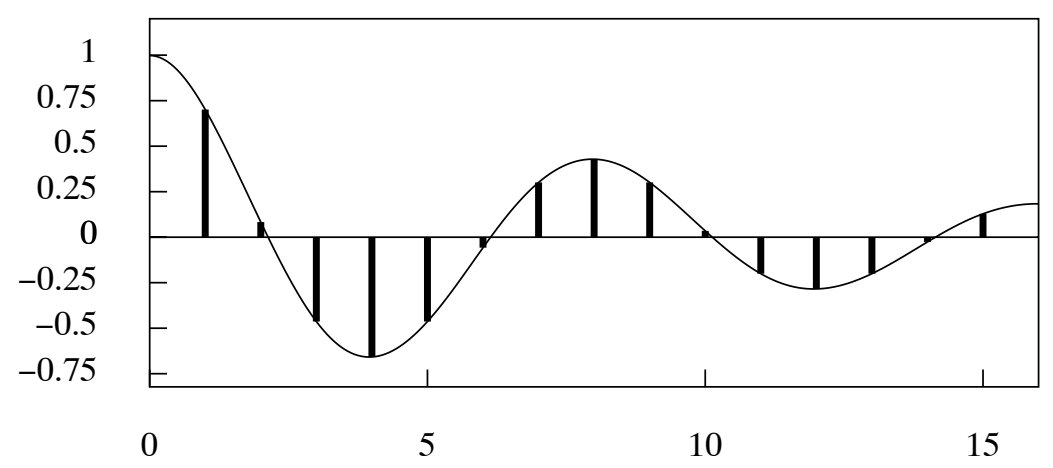

Fig. 5. A continuous autocovariance function of an $\mathrm{AR}(2)$ process, obtained via the inverse Fourier transform of the spectral density function, together with the corresponding discrete-time autocovariances, calculated from the AR parameters.

The spectrum of the discrete-time process, denoted by $f(\omega)$, is the Fourier transform of the sequence of autocovariances. It is a periodic function that is generated by the function $\gamma(z)$ when $z$ travels around the perimeter of the unit circle in the complex plane. When $z=\exp \{-\mathrm{i} \omega\}$ within $\gamma(z)$, the spectrum is generated as $\omega$ runs from $-\pi$ to $\pi$.

The spectrum of a continuous-time ARMA process, which is limited in frequency to the Nyquist interval $[-\pi, \pi]$, is no longer a periodic function. However, it has the same values over the interval as the periodic spectrum of the corresponding discrete-time process. The autocovariance function of the continuous-time process is given by the inverse Fourier integral transform of this spectrum. Thus

$$
\gamma(\tau)=\int_{-\pi}^{\pi} e^{\mathrm{i} \omega \tau} f(\omega) d \omega=\int_{0}^{\pi} 2 \cos (\omega \tau) f(\omega) d \omega
$$

where the second equality follows in consequence of the symmetry of $f(\omega)=$ $f(-\omega)$.

Since no tractable analytic expression is available for evaluating this integral exactly, it must be approximated via a discrete cosine Fourier transform:

$$
\gamma(\tau) \simeq \gamma_{N}^{\circ}(\tau)=\frac{2 \pi}{N} \sum_{j=0}^{[N / 2]} \cos \left(\omega_{j} \tau\right) f\left(\omega_{j}\right), \quad \omega_{j}=\frac{2 \pi j}{N}
$$

Here, $N$ is the number of points sampled from the function $f(\omega)$ over the interval $[-\pi, \pi]$, whereas $\gamma_{N}^{\circ}(\tau)$ is the circular autocovariance for samples of size $N$. It will be found is $\gamma_{N}^{\circ}(\tau) \rightarrow \gamma(\tau)$ as $N \rightarrow \infty$. However, the continuoustime autocovariances are generated more easily by allowing the index $\tau$ of equation (17) to vary continuously.

An example of the Fourier approximation of the autocovariance function is provided by Figure 5, where a sequence of $N=256$ elements sampled from the function $f(\omega)$ has been transformed to create a continuous piecewise linear 


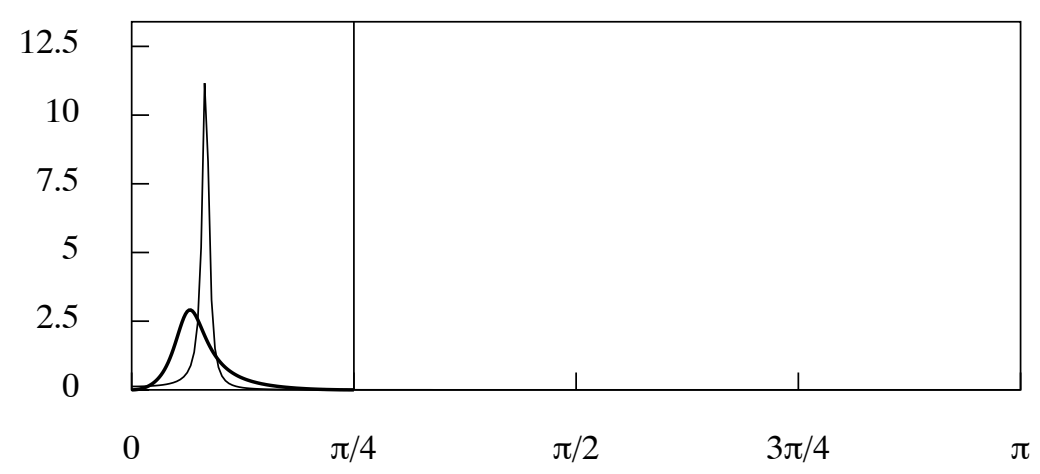

Fig. 6. The parametric spectrum of an $\operatorname{ARMA}(2,2)$ process, limited in frequency to $\pi$ radians per period and oversampled at the rate of 4 observations per period, represented by a heavy line, together with the parametric spectrum of an $\operatorname{AR}(2)$ model fitted to the sampled autocovariances.

rendition of $\gamma(\tau)$ over the range $\tau \in[0,16]$. The ordinates of the discrete-time autocovariance function are also plotted on the diagram. These have been obtained directly from the autoregressive parameters by the recursive method. Their coincidence with the ordinates of the continuous function testifies to the accuracy of the approximation.

\section{Consequences of Fitting ARMA Models to Oversampled Data}

Before describing the appropriate recourses for dealing with oversampled data, in which the maximum frequency is less than the Nyquist value of $\pi$ radians per sample interval, it is appropriate to analyse the effects that have been illustrated in section 2 .

From the theoretical autocovariance function of a continuous-time process supported on the Nyquist interval $[-\pi, \pi]$, it will be possible to derive the autocovariances of a discrete-time process that would be obtained by overrapid sampling. From these discrete theoretical autocovariances, one can infer the parameters of a misspecified ARMA model that represents the asymptotic limits of estimates based on oversampled data.

If the sampled values are separated by intervals of $\omega_{c} / \pi<1$ units of time, then the spectrum of the resulting discrete-time process will be supported on an interval $\left[-\omega_{c}, \omega_{c}\right]$, which is a subset of the Nyquist interval. Its spectrum will be zero-valued over the complementary interval within Nyquist interval, which has been described as a dead space.

Having sampled the autocovariances from the continuous function, a method of moments can be used to infer the corresponding parameters. In the case of a pure AR process, this is a matter of solving the Yule-Walker equations. In the case of an ARMA model, an iterative procedure is also required for finding the moving-average parameters via a Cramér-Wold factorisation. An effective procedure that exploits the Newton-Raphson algorithm has been expounded 


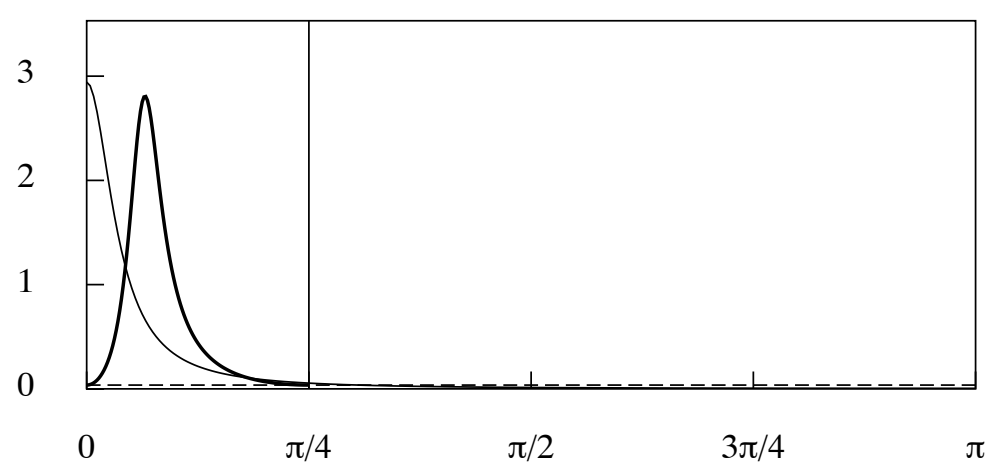

Fig. 7. The parametric spectrum of the oversampled $\operatorname{ARMA}(2,2)$ process, represented by a heavy line, supported on the spectrum of a white-noise contamination, together with the parametric spectrum of an $\operatorname{AR}(2)$ model fitted to the sampled autocovariances.

by Tunicliffe-Wilson (G.T. Wilson) (1969). The Yule-Walker procedure and the Cramér-Wold factorisation have been combined in a procedure, coded in both in $\mathrm{C}$ and in Pascal, that has been provided by Pollock (1999).

This procedure has been in incorporated in a program, OVERSAMPLE.PAS, that is available at the following web address:

$$
\text { http://www.le.ac.uk/users/dsgp1/ }
$$

Apart from determining the effects of oversampling, the program can be used to investigate the effects of a white-noise contamination of the data generated by the specified process.

Here, the program will be used to reaffirm what has already been revealed in Section 2 using an empirical data sequence. For the experiments, an $\operatorname{ARMA}(2,2)$ process is specified, which is regarded as the truth and which is designated Model (a). This is represented by the equation

$$
y(k)+\alpha_{1} y(k-1)+\alpha_{2} y(k-2)=\beta_{0} \varepsilon(t)+\beta_{1} \varepsilon(k-1)+\beta_{2} \varepsilon(k-2),
$$

where $\varepsilon(k)$ is a discrete-time white-noise process of unit variance. The autoregressive parameters are set to $\alpha_{1}=-1.0607$ and $\alpha_{2}=0.5625$. This is equivalent to specifying a pair of conjugate complex roots $\rho \exp \{ \pm \mathrm{i} \theta\}$ with a modulus $\rho=0.75$ and with arguments of $\pm \theta= \pm 45^{\circ}$. These values are recorded in Table 1 , which also displays some misspecified models. The moving-average parameters of Model (a) are $\beta_{0}=1, \beta_{1}=0$ and $\beta_{2}=-1$. Their effect is to place zeros on the unit circle at zero frequency and at the Nyquist frequency of $\pi$ radians per unit sample period. The zero at zero frequency mimics a characteristic of a detrended data sequence and the zero at $\pi$ emphasises the limitation of the process to the frequency interval $[0, \pi]$.

Figure 6 shows, via the heavy line, the parametric spectrum of the specified process when it has been subject to sampling at the rate of 4 observations per unit period, which confines the plotted spectrum to the frequency band $[0, \pi / 4]$. This oversampled version of Model (a) is designated Model (b). 
With this rate of sampling, the effective modulus of the autoregressive roots is $0.9306=(0.75)^{1 / 4}$ and their effective arguments are $\pm 11.25^{\circ}= \pm 45^{\circ} / 4$. The emptiness of the dead space in the interval $(\pi / 4, \pi]$, which signifies the absence of any noise contamination, can also be interpreted either as the result of the application to the data of a perfect low pass filter or as a consequence of a weighting scheme within the estimator that sets the spectral ordinates within this interval to zero.

The figure also shows the parametric spectrum of an $\mathrm{AR}(2)$ model derived from the autocovariances sampled at this rate from their continuous function, which is Model (c). It can be seen that the spectral spike of the model is excessively prominent and that its peak is at a higher frequency that the peak of the spectrum of the true process.

The estimated autoregressive parameters are $\alpha_{1}=-1.9062$ and $\alpha_{2}=$ 0.9735, which correspond to conjugate complex roots with a modulus $\rho=$ 0.9866 and with arguments of $\pm \theta= \pm 14.9873^{\circ}$. The polar parameters imply cyclical fluctuations that are both more rapid and more persistent than the actual fluctuations that would be generated by the true model.

Figure 7 shows the parametric spectrum of the oversampled process supported on the spectrum of a minimal white-noise contamination that extends over the entire frequency range of $[0, \pi]$ and which is represented by a broken line that is barely raised above the horizontal axis. This is to be construed as a discrete-time process that contaminates the individual observations, as opposed to a continuous background noise. The variance of the noise is 0.05 of the variance of the $\operatorname{ARMA}(2,2)$ process. The figure also shows the parametric spectrum of an $\operatorname{AR}(2)$ model that has been fitted to the autocovariances of the contaminated process.

The difference between the true spectrum and the spectrum of the fitted model, Model (d), is considerable. The fitted spectrum betrays the fact that the autoregressive polynomial of the model has a pair or real-valued roots in place of the conjugate complex roots of the process. The estimated autoregressive parameters are $\alpha_{1}=-1.0386$ and $\alpha_{2}=0.1291$ and the corresponding real-valued roots of the autoregressive polynomial are $\mu_{1}=0.1443$ and $\mu_{2}=0.8943$.

The results of these experiments can be explained by reference to the autocovariance function. When the rate of sampling is excessive, the autocovariances will be sampled at points that are too close to the origin, where the variance is to be found. Then, their values will decline at a diminished rate. This reduction in the rate of convergence is reflected in the modulus of the estimated complex roots, which indicates a rate of damping that understates the true value.

The opposite effect is experienced when there is a noise contamination. Then, the full variance of the noise will be added to the variance of the underlying process. Nothing will be added to the adjacent sampled ordinates of autocovariance function. Therefore, the sampled autocovariances will decline at an enhanced rate. If this rate of convergence exceeds the critical value, 
Table 1

The poles (autoregressive roots) of the true $\operatorname{ARMA}(2,2)$ model and of the $\operatorname{AR}(2)$ models fitted to the ordinates of the oversampled autocovariance function.

\begin{tabular}{lcccc}
\hline The Model & $\begin{array}{c}\text { Sampling } \\
\text { Interval }\end{array}$ & Poles & Modulus & $\begin{array}{c}\text { Argument } \\
\text { in Degrees }\end{array}$ \\
\hline $\begin{array}{l}\text { (a) True ARMA(2,2) } \\
\text { with unit sampling }\end{array}$ & 1.0 & $0.5303 \pm \mathrm{i} 0.5303$ & 0.75 & \pm 45.00 \\
\hline $\begin{array}{l}\text { (b) True ARMA(2,2) } \\
\text { with oversampling }\end{array}$ & 0.25 & $0.9127 \pm \mathrm{i} 0.1816$ & 0.9306 & \pm 11.25 \\
\hline $\begin{array}{l}\text { (c) Fitted AR(2) } \\
\text { with oversampling }\end{array}$ & 0.25 & $0.9531 \pm \mathrm{i} 0.2552$ & 0.9866 & \pm 14.99 \\
\hline $\begin{array}{l}\text { (d) Fitted AR(2) with } \\
\text { noise and oversampling }\end{array}$ & 0.25 & $0.1443,0.8943$ & - & - \\
\hline
\end{tabular}

then there will be a transition from cyclical convergence to monotonic convergence. In that case, the estimated autoregressive roots will be real-valued, which belies the complex dynamics of the true process, which would be liable to generate data with well-defined cycles.

\section{Estimation Methods for Oversampled Data}

The method that is proposed for estimating the parameters of an oversampled (frequency-limited) ARMA process relies on the possibility of resampling the data. The method entails the assumption that, underlying the sample, there is a trajectory generated by a continuous ARMA process. It is presumed that the limiting frequency within this process is $\omega_{c}<\pi$ and that the original sample has been taken at unit intervals. (This circumstance can be compared to that of the experiments of the previous section, where the limiting frequency of the process was $\pi$ radians per unit period and where 4 observation were assumed to be taken within that period. Both circumstances amount to oversampling.)

In view of the frequency limit of the process, it would have been appropriate to take the sample at the wider intervals of $\tau=\pi / \omega_{c}$. If $\tau$ is an integer number, then it will be sufficient to take one in every $\tau$ elements from the original sample and, thereafter, to estimate a discrete-time ARMA model in the usual way. If $\tau$ is not an integer, then the fact that the underlying continuous trajectory of the signal can be reconstituted from the original sample implies that synthetic sample points can be obtained that are separated by the requisite non-integer interval of $\pi / \omega_{c}$ units.

To derive such sample points, the Fourier transform must be taken of the original $T$ data points. This delivers the coefficients $\alpha_{j}, \beta_{j} ; j=0,1, \ldots,[T / 2]$, which are associated with the trigonometric functions at the Fourier frequencies $\omega_{j}=2 \pi j / T$. Let $q$ be the index such that $\omega_{q} \geq \omega_{c}$ is the least upper 


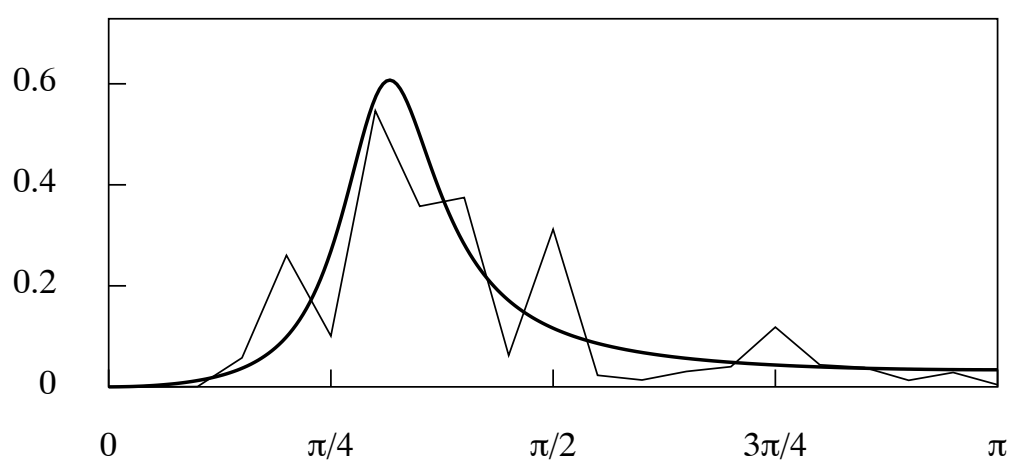

Fig. 8. The periodogram of the data that have been filtered and subsampled at the rate of 1 observation in 4 , overlaid by the parametric spectrum of an estimated $\operatorname{ARMA}(2,1)$ model.

bound of $\omega_{c}$ amongst the Fourier frequencies. Then, the formula that would be used to generate the reconstituted signal is

$$
x(t)=\sum_{j=0}^{q}\left\{\alpha_{j} \cos \left(\omega_{j} t\right)+\beta_{j} \sin \left(\omega_{j} t\right)\right\}
$$

This is just a truncated version of the summation of (9). It differs from that of (9) only by the omission of the coefficients $\alpha_{j}, \beta_{j}$ for $j>q$ that are deemed to be zero-valued. Whereas the continuous trajectory would be generated by allowing $t$ to vary continuously, the resampled data are generated by setting $t=\left(\pi / \omega_{c}\right) \times k$ for $k=0,1, \ldots,\left[T \omega_{c} / \pi\right]$.

The truncated summation of (21) excludes any elements of noise contamination that lie in the dead-space interval $\left(\omega_{c}, \pi\right]$. If such contamination is thought to be minor, then its presence within the interval $\left[0, \omega_{c}\right]$ can be safely ignored.

The noise contamination affecting an $\operatorname{AR}(p)$ process can often be captured by the moving-average component of an $\operatorname{ARMA}(p, q)$ model fitted to the data (The addition of a white-noise contamination to an $\operatorname{AR}(p)$ process gives rise to an $\operatorname{ARMA}(p, p)$ process.)

In applying the method of moments estimator to an $\operatorname{ARMA}(p, q)$ model, the empirical counterpart of the sequence of autocovariances $\gamma_{0}, \gamma_{1}, \ldots, \gamma_{q}$ is entailed in the calculation of the moving-average component, while that of the sequence $\gamma_{q+1-p}, \gamma_{q+2-p}, \ldots, \gamma_{q+p}$ is used in calculating the autoregressive parameters. (See, for example, Pollock 1999, p. 545.) If there is a pre-specified moving-average component of order $q$, then the autoregressive parameters alone are estimated from the latter sequence.

These strategies of estimation have been incorporated in a computer program, CONGRESS.PAS, that is available at the web address that has been provided in section 4 . A single zero at zero frequency may be imposed on the estimates by setting $\beta(z)=1-z$, and a zero at the Nyquist frequency $\pi$ can be imposed by setting $\beta(z)=1+z$. To impose zeros at both points requires 
setting $\beta(z)=1-z^{2}$. The program, which allows these choices, also contains procedures for generating pseudo-random data via frequency-limited ARMA processes.

The program has been applied to the data represented by Figure 1. An $\operatorname{ARMA}(2,1)$ model, with a zero at zero frequency, has been fitted to values sampled from this trajectory at $1 / 4$ of the original sample rate, which makes this an annual rate. In Figure 8, the parametric spectrum of the fitted model has been superimposed upon the periodogram of the subsampled data. It will be seen that this periodogram has a distended version the profile of the spectral structure that is supported of the interval $[0, \pi / 4]$ in Figures 2 and 3. Little is altered when an additional zero is imposed at $\pi$. The parametric spectrum fits the periodogram well.

It should be emphasised that the usual procedures of least-squares and maximum-likelihood estimation are applicable to the resampled data, regardless of their unusual provenance. In particular, the sample properties of the estimators are not affected and the usual forecasting methods continue to apply. However, the standard software that is available does not enable the imposition of pre-specified moving-average zeroes, which accounts of our use of an empirical method-of-moments estimator, which is a derivative of the Yule-Walker estimator.

An alternative approach to improving the performance of an AR model relies upon a weighted Whittle estimator. This approach had been pursued by various authors including, notably, Haywood and Tunnicliffe-Wilson, (1997) and Proietti (2008). However, it is a delicate matter to find a weighting scheme that will allow one to navigate between the opposing hazards that are represented by the two experiments of the previous section. Nevertheless, it has been shown that an appropriate weighting scheme can serve to enhance greatly the forecasting performance of an ARMA model.

Another method, which may have a similar inspiration to that of Haywood and Tunnicliffe-Wilson (1997), is due to Morton and Tunnicliffe-Wilson (2004). (See, also, Tunnicliffe-Wilson et al. 2016.) These authors propose a model that incorporates a lowpass filter that depends upon a single estimable parameter. The filter serves to attribute appropriate weights to the Fourier elements in the low-frequency range.

Finally, it should be acknowledged that the problems besetting oversampled processes will be alleviated by greatly increasing the autoregressive order in the estimated model and by including a moving-average component. However, given the proliferation of the autoregressive parameters and, therefore, of the poles of the transfer function, a simple characterisation of the cyclical dynamics of the signal will no longer be readily available. 


\section{Frequency-Limited Stochastic Differential Equations}

A description has been provided of a continuous frequency-limited trajectory, which is derived from the data points of a discrete-time ARMA process by sinc-function interpolation. It is possible to describe a stochastic differential equation that will generate such a trajectory and to obtain estimates of its parameters. This section describes methods for achieving this.

The conventional linear stochastic differential equation of orders $p$ and $q$, denoted by $\operatorname{LSDE}(p, q)$, is represented by

$$
\begin{aligned}
\left(\phi_{0} D^{p}+\phi_{1} D^{p-1}+\cdots+\phi_{p}\right) y(t) & =\left(\theta_{0} D^{q}+\theta_{1} D^{q-1}+\cdots+\theta_{q}\right) \zeta(t) \\
\text { or } \quad \phi(D) y(t) & =\theta(D) \zeta(t)
\end{aligned}
$$

wherein $D$ is the derivatives operator such that $D x(t)=d x(t) / d t$ and where $\zeta(t)$ represents the forcing function. The usual normalisation sets $\phi_{0}=1$, as would be the case of a second-order equation describing the acceleration of a body in a viscous medium that is subject to a random forcing function.

The forcing function $\zeta(t)$ of the LSDE model is commonly assumed to be constituted from the instantaneous and infinitesimal increments of a Wiener process, which are unbounded in frequency.

In the case of the continuous frequency-limited model, which bears a oneto-one correspondence with a discrete-time ARMA model, the forcing function $\varepsilon(t)$ is a frequency-limited white-noise process, supported on the Nyquist frequency interval $[-\pi, \pi]$, which can be constituted from a train of sinc functions.

Provided that $p>q$, and provided that the polynomial equation $\phi(s)=0$ does not contain repeated roots, the rational form of the $\operatorname{LSDE}(p, q)$ model is amenable to the following partial-fraction expansion:

$$
\begin{aligned}
y(t)=\frac{\theta(D)}{\phi(D)} \zeta(t) & =\left\{\frac{c_{1}}{D-\kappa_{1}}+\frac{c_{2}}{D-\kappa_{2}}+\cdots+\frac{c_{p}}{D-\kappa_{p}}\right\} \zeta(t) \\
& =\int_{0}^{\infty}\left\{c_{1} e^{\kappa_{1} \tau}+c_{2} e^{\kappa_{2} \tau}+\cdots+c_{p} e^{\kappa_{p} \tau}\right\} \zeta(t-\tau) d \tau .
\end{aligned}
$$

The discrete-time $\operatorname{ARMA}(p, q)$ model has an analogous partial-fraction expansion:

$$
\begin{aligned}
y(k)=\frac{\beta(L)}{\alpha(L)} \varepsilon(k) & =\left\{\frac{d_{1}}{1-\mu_{1} L}+\frac{d_{2}}{1-\mu_{2} L}+\cdots+\frac{d_{p}}{1-\mu_{p} L}\right\} \varepsilon(k) \\
& =\sum_{\tau=0}^{\infty}\left\{d_{1} \mu_{1}^{\tau}+d_{2} \mu_{2}^{\tau}+\cdots+d_{p} \mu_{2}^{\tau}\right\} \varepsilon(k-\tau) .
\end{aligned}
$$

Here, $L$ is the lag operator, which has the effect that $L \varepsilon(k)=\varepsilon(k-1)$ when applied to a sequence $\varepsilon(k)$.

The principle of impulse invariance is a common means of deriving the discrete-time counterpart of a continuous-time filter. The principle proposes 
that the discrete-time transfer function should be matched to the continuoustime function by equating the ordinates of the two impulse response functions at the sample points, such that

$$
\sum_{j} d_{j} \mu_{j}^{\tau}=\sum_{j} c_{j} e^{\kappa_{j} \tau} \quad \text { for } \quad \tau \in\{0,1,2, \ldots\}
$$

This can be achieved by setting $d_{j}=c_{j}$ and $\mu_{j}=\exp \left\{\kappa_{j}\right\}$ for $j=1,2, \ldots, p$. (For an exposition of the principle, see Oppenheim et al. 1983, Ch 7.)

The principle of impulse invariance can be used for deriving the continuoustime counterpart of a discrete-time model. Thus, If $\mu$ is a real-valued pole of the ARMA model, then $\kappa=\ln \mu$ is the corresponding pole of the LSDE model. If $\mu=\exp \{\kappa\}=\rho \exp \{\mathrm{i} \omega\}$ is complex-valued, and if it can be assumed that $\omega \in[-\pi, \pi]$, then $\kappa=\ln \mu=\ln \rho+\mathrm{i} \omega$. A necessary restriction on the ARMA pole is that $\mu>0$, if it is real-valued, or that $\rho>0$, if it is complex-valued

(It should be observed that, when the poles are complex-valued, the expression $\mu=\exp \{\kappa\}$ represents a many-to-one mapping from a set of continuoustime parameters to a discrete-time parameter. For, with $\kappa=\delta+\mathrm{i} \omega$, there is

$$
\mu=e^{\kappa}=e^{\delta} e^{\mathrm{i} \omega}=e^{\delta}\{\cos (\omega+2 \pi n)+\mathrm{i} \sin (\omega+2 \pi n)\},
$$

where $n$ is an arbitrary integer. Thus, in the absence of a restriction that sets $n=0$ and that confines $\omega$ to the Nyquist interval, there are many continuous processes which, when sampled at equal intervals, yield the same discrete process. This is the essence of the problem of aliasing.)

The differential equation that corresponds to the continuous ARMA process can be derived by compounding the partial fractions

$$
\frac{c_{j}}{D-\kappa_{j}}=\frac{d_{j}}{D-\ln \mu_{j}} ; \quad j=1, \ldots, p
$$

It will be seen that, if $q<p$ in the $\operatorname{ARMA}(p, q)$ model, then the result will be an $\operatorname{LSDE}(p, p-1)$ model.

Another method of deriving a continuous-time model as a counterpart to a discrete-time model is via the autocovariance principle. The principle proposes that the parameters of the continuous-time model should be determined by equating the continuous autocovariance function with the available discretetime function at the integer points over which the latter is defined.

For an continuous ARMA model driven by a frequency-limited whitenoise process supported on the Nyquist interval, the autocovariance function is provided by equation (17), when $\tau \in \mathcal{R}$ is a continuous index. In this case, the impulse invariance principle is equivalent to the autocovariance principle; and the derivation of the LSDE is straightforward.

In the case where it is assumed that the continuous model is driven by a process $\zeta(t)$ comprising the increments of a Wiener process, an alternative continuous autocovariance function is appropriate that is given by 


$$
\begin{aligned}
\gamma_{c}(\tau)=\sigma_{\zeta}^{2} \int_{0}^{\infty} \psi(t) \psi(t+\tau) d t & =\sigma_{\zeta}^{2} \sum_{i=1}^{p} \sum_{j=1}^{p}\left\{c_{i} c_{j} \int_{0}^{\infty} e^{\left(\kappa_{i}+\kappa_{j}\right) t+\kappa_{i} \tau} d t\right\} \\
& =\sigma_{\zeta}^{2} \sum_{i=1}^{p}\left\{\sum_{j=1}^{p} c_{i} c_{j} \frac{-e^{\kappa_{i} \tau}}{\kappa_{i}+\kappa_{j}}\right\} .
\end{aligned}
$$

The translation from the poles of the discrete-time model to those of the continuous model is unaffected. However, it is no longer appropriate to set $c_{j}=d_{j}$. Instead, the partial-fraction numerators must be determined by equating the discrete-time and the continuous-time autocovariances at the integer points, for which an iterative procedure is required,

One can also endeavour to estimate the continuous-time parameters directly from the data. The difficulty of such an approach is in the non-observability of the time derivatives of the data. Additional problems are posed when there is a moving-average component to contend with.

A template for the estimation of a second-order autoregressive $\operatorname{LSDE}(2,0)$ model from a continuous record has been provided by Priestley (1981, p. 382 ). The method is the continuous-time analogue of an ordinary least-squares estimation.

If it is assumed that the $\operatorname{LSDE}(2,0)$ model is driven by the increments of a Wiener process, then any sampled sequence of the forcing function will constitute a discrete-time white-noise process, regardless of the rate of sampling. The problem remains of how to obtain estimates of the derivatives at the sample points. If this can be achieved, then the parameters can be estimated efficiently by ordinary least-squares regression.

To obtain a discrete-time white-noise sequence from the continuous frequency-limited white-noise process defined in section 4 , it is necessary for the sampling to be at the Nyquist rate, which acquires that two observations in the time that it takes the element of highest frequency to complete a single cycle.

In that case, as the sampling theorem indicates, the continuous trajectory that underlies the data can be reconstituted by Fourier or sinc function interpolation. Since the resulting trajectory is an analytic function, is it possible to obtain derivatives of any order at each of the sample points. Therefore, the ordinary least-squares method of estimating a $\operatorname{LSDE}(p, 0)$ model in this way is entirely practical, albeit that it takes time to calculate the derivatives.

\section{References}

[1] Garnier, H., and L. Wang, L., 2008. Identification of Continuous-time Models from Sampled Data, Springer-Verlag, London.

[2] Haywood, J., and Tunnicliffe-Wilson, G., 1997. Fitting time series models by minimising multistep-head errors: a frequency domain approach, Journal of the Royal Statistical Society, Series B (Methodological), 59, 237-254. 
[3] Hodrick, R.J., and Prescott, E.C., 1980. Postwar U.S. business cycles: an empirical investigation, Working Paper, Carnegie-Mellon University, Pittsburgh, Pennsylvania.

[4] Hodrick R.J., and Prescott, E.C., 1997. Postwar U.S. business cycles: an empirical investigation, Journal of Money, Credit and Banking, 29, 1-16.

[5] Leser, C.E.V., 1961. A simple method of trend construction, Journal of the Royal Statistical Society, Series B, 23, 91-107.

[6] Luke, H.D., 1999. The origins of the sampling theorem, IEEE Communications Magazine, 37:4, 106-108.

[7] Morton, A.S., and Tunnicliffe-Wilson, G., 2004. A class of modified highorder autoregressive models with improved resolution of low frequency cycles, Journal of Time Series Analysis, 25, 235-250.

[8] Nyquist, H., 1924. Certain factors affecting telegraph speed, Bell System Technical Journal, 3, 324-346.

[9] Nyquist, H., 1928. Certain topics in telegraph transmission theory, Transactions of the AIEE, 47, 617-644. Reprinted in 2002, Proceedings of the IEEE, 90, 280-305.

[10] Oppenheim, A.V., Willsky, A.S., and Young, I.T., 1983. Signals and Systems, Prentice-Hall International, London.

[11] Pagan, A., 1997. Towards an understanding of some business cycle characteristics, The Australian Economic Review, 30, 1-15.

[12] Pandit, S.M., and Wu, S-M., 1983. Time Series and System Analysis with Applications, Krieger Publishing Co., Malabar, Florida.

[13] Pollock, D.S.G., 1999. A Handbook of Time-Series Analysis, Signal Processing and Dynamics, Academic Press, London.

[14] Pollock, D.S.G., 2009. Realisations of finite-sample frequency-selective filters, Journal of Statistical Planning and Inference, 139, 1541-1558.

[15] Priestley, M.B., 1981. Spectral Analysis of Time Series, Volume 1., Academic Press, London.

[16] Proietti, T., 2008. Band spectral estimation for signal extraction, Economic Modelling, 25, 54-69.

[17] Shannon, C.E., 1949. Communication in the presence of noise, Proceedings of the Institute of Radio Engineers, 37, 10-21. Reprinted in 1998, Proceedings of the IEEE, 86, 447-457.

[18] Tunnicliffe-Wilson, G., Reale, M., and Haywood, J., 2016. Models for Dependent Time Series, CRC Press, Boca Raton, Florida.

[19] Wilson, G.T., 1969. Factorisation of the covariance generating fFunction of a pure moving average process, SIAM Journal of Numerical Analysis, 6, 1-7. 